International Mathematical Forum, 4, 2009, no. 15, 727 - 732

\title{
On Additive Mappings in Rings with Identity Element
}

\author{
Basudeb Dhara \\ Department of Mathematics, Belda College, Belda \\ Paschim Medinipur-721424, India \\ basu_dhara@yahoo.com
}

R. K. Sharma

Department of Mathematics, Indian Institute of Technology, Delhi

Hauz Khas, New Delhi-110016, India

rksharma@maths.iitd.ernet.in

\begin{abstract}
Let $R$ be an associative ring with identity element, $F: R \rightarrow R$, $D: R \rightarrow R$ and $T: R \rightarrow R$ all additive mappings and $n \geq 1$ a fixed integer. We prove that (i) if $R$ is $(n+1)$ !-torsion free any ring such that $F\left(x^{n+1}\right)=F(x) x^{n}+x D(x) x^{n-1}+x^{2} D(x) x^{n-2}+\cdots+x^{n} D(x)$ holds for all $x \in R$, then $D$ is a Jordan derivation and $F$ is a Jordan generalized derivation; (ii) if $R$ is a $(n+2)$ !-torsion free semiprime ring such that $(n+1) T\left(x^{n+1}\right)=x^{n} T(x)+x^{n-1} T(x) x+\cdots+T(x) x^{n}$ holds for all $x \in R$, then there exists $a \in Z(R)$ such that $T(x)=a x$ for all $x \in R$.
\end{abstract}

Mathematics Subject Classification: 16W25, 16R50, 16N60

Keywords: Semiprime ring, derivation, generalized derivation

In this article $R$ denotes an associative ring. A ring $R$ is called $n$-torsion free if $n x=0$ for $x \in R$ implies that $x=0$. An additive mapping $D$ from $R$ to $R$ is called a derivation if $D(x y)=D(x) y+x D(y)$ for all $x, y \in R$, and is called a Jordan derivation if $D\left(x^{2}\right)=D(x) x+x D(x)$ holds for all $x \in R$. Clearly, every derivation is a Jordan derivation. The converse is not true in general. A well known result of Herstein [5] states that every Jordan derivation on a prime ring of characteristic different from two is a derivation. Brešar and Vukman proved this result briefly in [3]. Further, Cusack [4] has

\footnotetext{
${ }^{0}$ This work is supported by a grant from University Grants Commission, India.
} 
generalized this result for semiprime ring stating that every Jordan derivation of a 2-torsion free semiprime ring is a derivation.

An additive mapping $T: R \rightarrow R$ is said to be a left (right) centralizer if $T(x y)=T(x) y($ respectively $T(x y)=x T(y))$ holds for all $x, y \in R$ and Jordan left (right) centralizer if $T\left(x^{2}\right)=T(x) x$ (correspondingly $T\left(x^{2}\right)=x T(x)$ ) holds for all $x \in R$. An additive mapping which is both left and right centralizer is called simply a centralizer mapping. Obviously, every left (right) centralizer mapping is a Jordan left (right) centralizer mapping, but the converse is not true in general. Zalar [10] has proved that every Jordan left (right) centralizer mapping in a 2-torsion free semiprime ring is a left (right) centralizer mapping.

In [2], Brešar has introduced the generalized derivation mapping which covers both the concepts of derivation and left centralizer mapping. An additive mapping $F: R \rightarrow R$ is called a generalized derivation if there exists a derivation $D: R \rightarrow R$ such that $F(x y)=F(x) y+x D(y)$ holds for all $x, y \in R$. An additive mapping $F: R \rightarrow R$ is said to be a Jordan generalized derivation if there exists a Jordan derivation $D: R \rightarrow R$ such that $F\left(x^{2}\right)=F(x) x+x D(x)$ holds for all $x \in R$. In [1], Ashraf and Rehman showed that in a 2-torsion free ring $R$ which has a commutator nonzero divisor, every Jordan generalized derivation on $R$ is a generalized derivation. Recently, Vukman [9] has proved that every generalized Jordan derivation on a 2-torsion free semiprime ring is a generalized derivation. In the present paper, our aim is to improve above results by considering the identity $F\left(x^{n+1}\right)=F(x) x^{n}+x D(x) x^{n-1}+x^{2} D(x) x^{n-2}+\cdots+x^{n} D(x)$ for all $x \in R$, where $R$ is any ring with identity element and $F, D$ are two additive mappings. More precisely, we prove the following:

Theorem 1. Let $n \geq 1$ be a fixed integer and let $R$ be a $(n+1)$ !-torsion free any ring with identity element. If $F: R \rightarrow R$ and $D: R \rightarrow R$ are two additive mappings such that $F\left(x^{n+1}\right)=F(x) x^{n}+x D(x) x^{n-1}+x^{2} D(x) x^{n-2}+$ $\cdots+x^{n} D(x)$ holds for all $x \in R$, then $D$ is a Jordan derivation and $F$ is a Jordan generalized derivation.

In [8], Vukman and Kosi-Ulbl proved that if $R$ is a 2-torsion free semiprime rings with extended centroid $C$ and $T: R \rightarrow R$ is an additive mapping such that $3 T(x y x)=T(x) y x+x T(y) x+x y T(x)$ holds for all $x, y \in R$, then there exists an element $\lambda \in C$ such that $T(x)=\lambda x$ for all $x \in R$. Recently, KosiUlbl [6] showed that if $R$ is a $(m+n+2)$ !-torsion free semiprime ring with the identity element, where $m \geq 1, n \geq 1$ are fixed integers, and $T: R \rightarrow R$ is an additive mapping such that $T\left(x^{m+n+1}\right)=x^{m} T(x) x^{n}$ holds for all $x \in R$, then $T$ is a centralizer mapping.

These results of Vukman and Kosi-Ulbl motivate us to consider the situation when $(n+1) T\left(x^{n+1}\right)=x^{n} T(x)+x^{n-1} T(x) x+\cdots+T(x) x^{n}$ for all $x \in R$, where $R$ is a semiprime ring with identity element and $T$ is an additive 
mapping. More precisely, we prove the following:

Theorem 2. Let $n>0$ be a fixed integer and let $R$ be $a(n+2)$ !-torsion free semiprime ring with identity element. If $T: R \rightarrow R$ is an additive mapping such that $(n+1) T\left(x^{n+1}\right)=x^{n} T(x)+x^{n-1} T(x) x+\cdots+T(x) x^{n}$ for all $x \in R$, then there exists $a \in Z(R)$ such that $T(x)=$ ax for all $x \in R$.

Proof of Theorem 1. We have the relation

$$
\begin{aligned}
F\left(x^{n+1}\right) & =F(x) x^{n}+x D(x) x^{n-1}+x^{2} D(x) x^{n-2}+\cdots+x^{n} D(x) \\
& =F(x) x^{n}+\sum_{i=1}^{n} x^{i} D(x) x^{n-i}
\end{aligned}
$$

for all $x \in R$. Let $e$ be the identity element of $R$, then replacing $x$ by $e$ in (1), we get $F(e)=F(e)+n D(e)$ which implies $n D(e)=0$. Since $R$ is $n$-torsion free, we may conclude that $D(e)=0$.

Now replacing $x$ by $x+k e$ in (1), where $k$ is any positive integer, we get

$$
F\left((x+k e)^{n+1}\right)=F(x+k e)(x+k e)^{n}+\sum_{i=1}^{n}(x+k e)^{i} D(x+k e)(x+k e)^{n-i}
$$

for all $x \in R$. Expanding the power values of $(x+k e)$ and using the fact $D(e)=0$, we have

$$
\begin{gathered}
F\left[x^{n+1}+\left(\begin{array}{c}
n+1 \\
1
\end{array}\right) k x^{n}+\cdots+\left(\begin{array}{c}
n+1 \\
n-1
\end{array}\right) k^{n-1} x^{2}+\left(\begin{array}{c}
n+1 \\
n
\end{array}\right) k^{n} x+k^{n+1} e\right] \\
=(F(x)+k F(e))\left\{x^{n}+\cdots+\left(\begin{array}{c}
n \\
n-2
\end{array}\right) k^{n-2} x^{2}+\left(\begin{array}{c}
n \\
n-1
\end{array}\right) k^{n-1} x+k^{n} e\right\} \\
+\sum_{i=1}^{n}\left\{x^{i}+\cdots+\left(\begin{array}{c}
i \\
i-2
\end{array}\right) k^{i-2} x^{2}+\left(\begin{array}{c}
i \\
i-1
\end{array}\right) k^{i-1} x+k^{i} e\right\} D(x) \\
\left\{x^{n-i}+\cdots+\left(\begin{array}{c}
n-i \\
n-i-2
\end{array}\right) k^{n-i-2} x^{2}+\left(\begin{array}{c}
n-i \\
n-i-1
\end{array}\right) k^{n-i-1} x+k^{n-i} e\right\}
\end{gathered}
$$

for all $x \in R$. Using relation (1), this can be written as

$$
k f_{1}(x, e)+k^{2} f_{2}(x, e)+\cdots+k^{n} f_{n}(x, e)=0
$$

for all $x \in R$. Now, replacing $k$ by $1,2,3, \ldots, n$ in turn, and considering the resulting system of $n$ homogeneous equations, we see that the coefficient matrix of the system is a Van der Monde matrix

$$
\left(\begin{array}{ccccc}
1 & 1 & 1 & \cdots & 1 \\
2 & 2^{2} & 2^{3} & \cdots & 2^{n} \\
\vdots & \vdots & \vdots & & \vdots \\
n & n^{2} & n^{3} & \cdots & n^{n}
\end{array}\right)
$$


Since the determinant of the matrix is equal to a product of positive integers, each of which is less than $n$, and since $R$ is $(n+1)$ !-torsion free, it follows immediately that $f_{i}(x, e)=0$ for all $x \in R, i=1, \ldots, n$. Now, $f_{n}(x, e)=0$ implies that

$$
(n+1) F(x)=F(x)+n F(e) x+n D(x)
$$

that is $n F(x)=n F(e) x+n D(x)$. Since $R$ is $n$-torsion free, $F(x)=F(e) x+$ $D(x)$ for all $x \in R$. Now, $f_{n-1}(x, e)=0$ gives

$$
\left(\begin{array}{c}
n+1 \\
2
\end{array}\right) F\left(x^{2}\right)=n F(x) x+\left(\begin{array}{c}
n \\
2
\end{array}\right) F(e) x^{2}+\sum_{i=1}^{n}\{i x D(x)+(n-i) D(x) x\} .
$$

Multiplying both sides by 2 , it reduces to

$$
\begin{aligned}
n(n+1) F\left(x^{2}\right)= & 2 n F(x) x+n(n-1) F(e) x^{2} \\
& +n(n+1) x D(x)+n(n-1) D(x) x .
\end{aligned}
$$

Since $R$ is $n$-torsion free, this gives

$$
\begin{aligned}
(n+1) F\left(x^{2}\right)= & 2 F(x) x+(n-1) F(e) x^{2} \\
& +(n+1) x D(x)+(n-1) D(x) x .
\end{aligned}
$$

Using $F(x)=F(e) x+D(x)$, this implies

$$
\begin{aligned}
(n+1)\left\{F(e) x^{2}+D\left(x^{2}\right)\right\}= & 2\left\{F(e) x^{2}+D(x) x\right\}+(n-1) F(e) x^{2} \\
& +(n+1) x D(x)+(n-1) D(x) x
\end{aligned}
$$

which is $(n+1) D\left(x^{2}\right)=(n+1)(D(x) x+x D(x))$ for all $x \in R$. Again, since $R$ is $(n+1)$-torsion free, we have $D\left(x^{2}\right)=D(x) x+x D(x)$ for all $x \in R$ which means $D$ is a Jordan derivation in $R$. Therefore, from $F(x)=F(e) x+D(x)$, we obtain that $F\left(x^{2}\right)=F(e) x^{2}+D(x) x+x D(x)=F(x) x+x D(x)$ for all $x \in R$, implying $F$ a Jordan generalized derivation in $R$. Thus the proof is complete.

Proof of Theorem 2. We have the relation

$$
\begin{aligned}
(n+1) T\left(x^{n+1}\right) & =x^{n} T(x)+x^{n-1} T(x) x+\cdots+T(x) x^{n} \\
& =\sum_{i=0}^{n} x^{i} T(x) x^{n-i}
\end{aligned}
$$

for all $x \in R$. Let $e$ be the identity element in $R$. Now replacing $x$ by $x+k e$ in (6), where $k$ is any positive integer, we get

$$
\begin{gathered}
(n+1) T\left[x^{n+1}+\left(\begin{array}{c}
n+1 \\
1
\end{array}\right) k x^{n}+\cdots+\left(\begin{array}{c}
n+1 \\
n-1
\end{array}\right) k^{n-1} x^{2}+\left(\begin{array}{c}
n+1 \\
n
\end{array}\right) k^{n} x+k^{n+1} e\right] \\
=\sum_{i=0}^{n}\left\{x^{i}+\cdots+\left(\begin{array}{c}
i \\
i-2
\end{array}\right) k^{i-2} x^{2}+\left(\begin{array}{c}
i \\
i-1
\end{array}\right) k^{i-1} x+k^{i} e\right\} T(x+k e) \\
\left\{x^{n-i}+\cdots+\left(\begin{array}{c}
n-i \\
n-i-2
\end{array}\right) k^{n-i-2} x^{2}+\left(\begin{array}{c}
n-i \\
n-i-1
\end{array}\right) k^{n-i-1} x+k^{n-i} e\right\}
\end{gathered}
$$


for all $x \in R$, where we denote $\left(\begin{array}{l}n \\ k\end{array}\right)=0$ for $k<0$ and for $k>n$. Using relation (6), this can be written as

$$
k f_{1}(x, e)+k^{2} f_{2}(x, e)+\cdots+k^{n} f_{n}(x, e)=0
$$

for all $x \in R$. Then by the similar argument as in the proof of Theorem 1, we have that $f_{i}(x, e)=0$ for all $x \in R, i=1, \ldots, n$. Now, $f_{n}(x, e)=0$ implies,

$$
(n+1)\left(\begin{array}{c}
n+1 \\
n
\end{array}\right) T(x)=\sum_{i=0}^{n}\left\{T(x)+\left(\begin{array}{c}
i \\
i-1
\end{array}\right) x T(e)+\left(\begin{array}{c}
n-i \\
n-i-1
\end{array}\right) T(e) x\right\}
$$

which gives multiplying both sides by 2 that

$$
2(n+1)^{2} T(x)=2(n+1) T(x)+n(n+1)(x T(e)+T(e) x) .
$$

Since $R$ is $(n+1)$-torsion free,

$$
2(n+1) T(x)=2 T(x)+n(x T(e)+T(e) x)
$$

which is $2 n T(x)=n(x T(e)+T(e) x)$. Again, since $R$ is $n$-torsion free, we obtain $2 T(x)=x T(e)+T(e) x$ for all $x \in R$. Now, $f_{n-1}(x, e)=0$ gives

$$
\begin{gathered}
(n+1)\left(\begin{array}{c}
n+1 \\
2
\end{array}\right) T\left(x^{2}\right)=\sum_{i=0}^{n}\left\{\left(\begin{array}{c}
i \\
i-1
\end{array}\right) x T(x)+\left(\begin{array}{c}
n-i \\
n-i-1
\end{array}\right) T(x) x\right. \\
\left.+\left(\begin{array}{c}
i \\
i-2
\end{array}\right) x^{2} T(e)+\left(\begin{array}{c}
n-i \\
n-i-2
\end{array}\right) T(e) x^{2}+\left(\begin{array}{c}
i \\
i-1
\end{array}\right)\left(\begin{array}{c}
n-i \\
n-i-1
\end{array}\right) x T(e) x\right\} \\
=\sum_{i=0}^{n}\left\{i(x T(x)+T(x) x)+\left(\begin{array}{c}
i \\
2
\end{array}\right)\left(x^{2} T(e)+T(e) x^{2}\right)+i(n-i) x T(e) x\right\} .
\end{gathered}
$$

Since, $\sum_{i=0}^{n}\left(\begin{array}{l}i \\ 2\end{array}\right)=\sum_{i=0}^{n} i(n-i)=\frac{(n+1) n(n-1)}{6}$, multiplying both sides by 6 in $(9)$, we have

$$
\begin{aligned}
3(n+1)^{2} n T\left(x^{2}\right)= & 3 n(n+1)(x T(x)+T(x) x) \\
& +(n+1) n(n-1)\left\{x^{2} T(e)+x T(e) x+T(e) x^{2}\right\} .
\end{aligned}
$$

Since $R$ is $(n+2)$ !-torsion free,

$$
\begin{aligned}
3(n+1) T\left(x^{2}\right)= & 3(x T(x)+T(x) x) \\
& +(n-1)\left\{x^{2} T(e)+x T(e) x+T(e) x^{2}\right\} .
\end{aligned}
$$

Now using $2 T(x)=x T(e)+T(e) x,(10)$ implies,

$$
\begin{aligned}
3(n+1)\left(x^{2} T(e)+T(e) x^{2}\right)= & 3 x(x T(e)+T(e) x)+3(x T(e)+T(e) x) x \\
& +2(n-1)\left\{x^{2} T(e)+x T(e) x+T(e) x^{2}\right\} .
\end{aligned}
$$

By simple manipulation, this reduces to $(n+2)[[T(e), x], x]=0$ for all $x \in R$. Since $R$ is $(n+2)$-torsion free, $[[T(e), x], x]=0$ for all $x \in R$. Since $R$ is semiprime ring, by [7, Theorem 2], $T(e) \in Z(R)$. Thus (??) implies that, $T(x)=T(e) x$, as desired. 


\section{References}

[1] M. Ashraf and N. Rehman, On Jordan generalized derivations in rings, Math. J. Okayama Univ. 42 (2000), 7-9.

[2] M. Brešar, On the distance of the composition of two derivations to the generalized derivation, Glasgow Math. J. 33 (1991), 89-93.

[3] M. Brešar and J. Vukman, Jordan derivations on prime rings, Bull. Aust. Math. Soc. 37 (1988), 321-324.

[4] J. M. Cusack, Jordan derivations on rings, Proc. Amer. Math. Soc. 53 (2) (1975), 321-324.

[5] I. N. Herstein, Jordan derivations of prime rings, Proc. Amer. Math. Soc. 8 (1957), 1104-1110.

[6] I. Kosi-Ulbl, A remark on centralizers in semiprime rings, Glasnik Matematicki, 39 (1) (2004), 21-26.

[7] C. Lanski, An Engel condition with derivation for left ideals, Proc. Amer. Math. Soc. 125 (2) (1997), 339-345.

[8] J. Vukman and I. Kosi-Ulbl, An equation related to centralizers in semiprime rings, Glasnik Matematicki, 38 (2003), 253-261.

[9] J. Vukman, A note on generalized derivations of semiprime rings, Taiwanese J. Math. 11 (2) (2007), 367-370.

[10] B. Zalar, On centralizers of semiprime rings, Comment. Math. Univ. Carol. 32 (1991), 609-614.

Received: August, 2008 